\title{
Experimental study on dynamic buckling phenomena for supercavitating underwater vehicle
}

\author{
Minho Chung ${ }^{1}$, Hee Jun Lee ${ }^{1}$, Yeon Cheol Kang ${ }^{1}$, Woo-Bin Lim ${ }^{1}$, Jeong Ho Kim ${ }^{1}$ \\ Jin Yeon Cho ${ }^{1}$, Wanil Byun ${ }^{2}$, Seung Jo Kim² and Sung-Han Park ${ }^{3}$ \\ ${ }^{1}$ Department of Aerospace Engineering, Inha University, Incheon, Korea \\ ${ }^{2}$ School of Mechanical and Aerospace Engineering, Seoul National University, Seoul, Korea \\ ${ }^{3}$ Agency for Defence Development, Daejeon, Korea
}

\begin{abstract}
Dynamic buckling, also known as parametric resonance, is one of the dynamic instability phenomena which may lead to catastrophic failure of structures. It occurs when compressive dynamic loading is applied to the structures. Therefore it is essential to establish a reliable procedure to test and evaluate the dynamic buckling behaviors of structures, especially when the structure is designed to be utilized in compressive dynamic loading environment, such as supercavitating underwater vehicle. In the line of thought, a dynamic buckling test system is designed in this work. Using the test system, dynamic buckling tests including beam, plate, and stiffened plate are carried out, and the dynamic buckling characteristics of considered structures are investigated experimentally as well as theoretically and numerically.
\end{abstract}

KEY WORDS: Dynamic buckling; Parametric resonance; Dynamic compressive load; Supercavitation.

\section{INTRODUCTION}

When a vehicle moves very fast under water, a large cavity may be generated and wrap the whole body of vehicle. This kind of phenomena is called supercavitation (Savchenko, 2001; Vlasenko, 2003; Hargrove, 2004; Vanek, Bokor, Balas and Arndt, 2007). The cavity around the vehicle body makes it possible to reduce the skin friction drastically between an underwater vehicle and water. Thus, underwater vehicle may travel under water in very high speeds, once a large cavity encompassing the whole body is generated. However, if a vehicle moves under water in high speeds, the underwater vehicle undergoes high compressive static and dynamic loads due to the thrust and pressure drag as shown in Fig. 1, which may cause dynamic buckling, also known as parametric resonance, of vehicle structure.

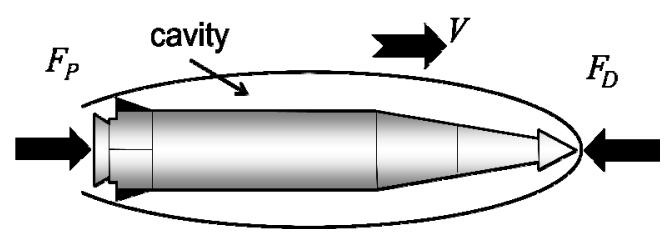

Fig. 1 Supercavitation phenomena and compressive loadings in high-speed under water vehicle.

Corresponding author: Jin Yeon Cho

e-mail:cjy@inha.ac.kr 
The dynamic buckling (parametric resonance) is one of the fatal structural instability phenomena, which is caused by dynamically oscillating compressive loads. Once the instability is initiated, a large transverse motion is generated in the structure, and as a result fatal damage may occur in the vehicle structure. Physics behind the dynamic buckling is similar to the parametric roll resonance of ship (Chang, 2008).

Therefore, dynamic buckling of vehicle structure should be considered in design of high speed underwater vehicle, and it is essential to construct reliable dynamic buckling test system to develop high-speed underwater vehicle. Regarding dynamic buckling (parametric resonance) theory, one can resort to references (Ahn and Ruzzene, 2006; Bolotin, 1964; Iwatsubo and Saigo, 1974; Park and Kim, 2002; Popov, 2003). However, regarding how to construct dynamic buckling test system, the issue is not sufficiently dealt with, although there are some references (Chen and Yeh, 2001; Deolasi and Datta, 1997; Langthjem and Sugiyama, 2000). In the line of thought, a dynamic buckling test system is developed and its validity and conceptual usefulness are investigated in this work. To design dynamic buckling test system, dynamic buckling is theoretically investigated at first. Based on the theoretical investigation, dynamic buckling test system is designed. Dynamic load is applied to the structure of interest through the coil spring with servo motor, and dynamic load cell is utilized to measure the applied dynamic load precisely. Static load is applied through lever with balance weight, and the interference between the structure and balance weight lever system is minimized through a low stiffness plate-type spring.

Regarding beam type structure, experimental studies are carried out by using the developed dynamic buckling test system, and the results are crosschecked with analytical solution. Further, experiments for plate and stiffened plate are carried out, and the experimental results are investigated and crosschecked with numerical solutions which are obtained by in-house program for dynamic buckling analysis (Byun, et al., 2011).

\section{THEORY OF DYNAMIC BUCKLING}

As mentioned in introduction, dynamic buckling may occur when compressive static and dynamic loads are applied to structures. In case of beam structures, the situation is sketched in Fig. 2.

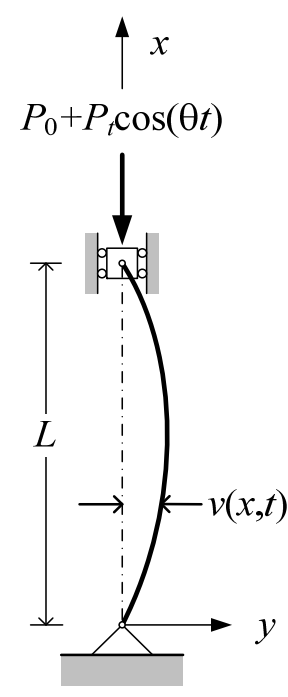

Fig. 2 Euler beam under static and dynamic compressive loadings.

For Euler beam, the equation of motion under compressive static and dynamic loads can be written as follows (Bolotin, 1964).

$$
\rho \frac{\partial^{2} v(x, t)}{\partial t^{2}}+E I \frac{\partial^{4} v(x, t)}{\partial x^{4}}+\left[P_{0}+P_{t} \cos (\theta t)\right] \frac{\partial^{2} v(x, t)}{\partial x^{2}}=0
$$


where $x$ and $t$ denote spatial coordinate and time. $\rho, E$, and $I$ correspond to density per unit length, Young's modulus, and area moment of inertia, respectively. $P_{0}$ is static compressive loading, and $P_{t}$ is amplitude of dynamic axial loading. $\theta$ is excitation frequency of dynamic axial loading, and period $T$ of dynamic load is $2 \pi / \theta$.

By separation of variables as shown in expression (2), the equation of motion can be changed into Eq. (3).

$$
\begin{gathered}
v(x, t)=\sum_{k=1}^{\infty} f_{k}(t) \sin \frac{k \pi x}{L} \\
\sum_{k=1}^{\infty}\left[\rho \frac{d^{2} f_{k}(t)}{d t^{2}}+E I \frac{k^{4} \pi^{4}}{L^{4}} f_{k}(t)-\left[P_{0}+P_{t} \cos (\theta t)\right] \frac{k^{2} \pi^{2}}{L^{2}} f_{k}(t)\right] \sin \frac{k \pi x}{L}=0
\end{gathered}
$$

It is noted that the expression (2) satisfies the boundary conditions of the problem of interest. To satisfy Eq. (3), the coefficient of each sine function should be zero for all $k$. Therefore Eq. (4) should be satisfied for all $k$.

$$
0=\rho \frac{d^{2} f_{k}(t)}{d t^{2}}+E I \frac{k^{4} \pi^{4}}{L^{4}} f_{k}(t)-\left[P_{0}+P_{t} \cos (\theta t)\right] \frac{k^{2} \pi^{2}}{L^{2}} f_{k}(t) \quad(k=1,2,3, \cdots)
$$

If one introduces natural frequency $\omega_{k}$ and critical Euler buckling load $P_{k}^{c r}$ of Euler beam as shown in Eq. (6), one can rewrite Eq. (4) as Eq. (5).

$$
\frac{d^{2} f_{k}(t)}{d t^{2}}+\omega_{k}^{2}\left(1-\frac{P_{0}+P_{t} \cos (\theta t)}{P_{k}^{c r}}\right) f_{k}(t)=0 \quad(k=1,2,3 \cdots)
$$

where

$$
\begin{aligned}
& \omega_{k}=\frac{k^{2} \pi^{2}}{L^{2}} \sqrt{\frac{E I}{\rho}} \\
& P_{k}^{c r}=\frac{k^{2} \pi^{2}}{L^{2}} E I
\end{aligned}
$$

Further, one can represent Eq. (5) in the form of Eq. (7) by introducing newly defined parameters $\Omega_{k}$ and $\mu_{k}$.

$$
\frac{d^{2} f_{k}(t)}{d t^{2}}+\Omega_{k}{ }^{2}\left[1-2 \mu_{k} \cos (\theta t)\right] f_{k}(t)=0 \quad(k=1,2,3 \cdots)
$$

where $\Omega_{k}$ is the natural frequency of beam subjected to constant compressive load $P_{0}$, and $\mu_{k}$ is excitation parameter as denoted in Eq. (8).

$$
\Omega_{k}^{2} \equiv \omega_{k}^{2} \frac{\left(P_{k}^{c r}-P_{0}\right)}{P_{k}^{c r}}
$$




$$
\mu_{k} \equiv \frac{P_{t}}{2\left(P_{k}^{c r}-P_{0}\right)}
$$

Additionally, if one considers only the fundamental mode $(k=1)$, then one can obtain well-known Mathieu-Hill equation (9) where subscript ' 1 ' is not denoted for convenience.

$$
\begin{gathered}
\frac{d^{2} f(t)}{d t^{2}}+\Omega^{2}[1-2 \mu \cos (\theta t)] f(t)=0 \\
\Omega^{2}=\left(\frac{\pi^{2}}{L^{2}} \sqrt{\frac{E I}{\rho}}\right)^{2}\left[1-P_{0} \frac{L^{2}}{\pi^{2} E I}\right], \mu=\frac{P_{t}}{2\left[\pi^{2} E I / L^{2}-P_{0}\right]}
\end{gathered}
$$

It is known that at least one of the solutions of Mathieu-Hill equation becomes periodic solution with period $T=2 \pi / \theta$ (or period $2 T=2 \times 2 \pi / \theta$ ) for certain combination of static/ dynamic loads and excitation frequency $\left(P_{0}, P_{t}, \theta\right)$. If the periodic motion at both ends of an interval in the parameter plane $(\theta /(2 \Omega), \mu)$ has the same period $T$ (or $2 T)$, then the motion for the parameters in the interval becomes unstable (Meirovitch, 1998). Further, the motion for interval in the parameter plane is most dangerous when the motions of both ends of the interval have the same period $2 T$. This region is called principal region of dynamic instability (Bolotin, 1964). To find the principal region of dynamic instability, the following form (11) with period $2 T(=2 \times 2 \pi / \theta)$ is assumed.

$$
f(t)=a \sin \frac{\theta t}{2}+b \cos \frac{\theta t}{2}
$$

Substitution of (11) into Eq. (9) and Fourier series expansion yield Eq. (12) after neglecting higher harmonic terms.

$$
0 \approx a \sin \frac{\theta t}{2}\left[-\frac{\theta^{2}}{4}+\Omega^{2}+\mu \Omega^{2}\right]+b \cos \frac{\theta t}{2}\left[-\frac{\theta^{2}}{4}+\Omega^{2}-\mu \Omega^{2}\right]
$$

From the above equation, non-trivial solution with period $2 T$ can be obtained if one of the conditions presented in Eq. (13) is satisfied.

$$
\left\{\begin{array}{l}
-\frac{\theta^{2}}{4}+\Omega^{2}+\mu \Omega^{2}=0 \text { and } b=0 \\
\text { or } \\
-\frac{\theta^{2}}{4}+\Omega^{2}-\mu \Omega^{2}=0 \text { and } a=0
\end{array}\right.
$$

Therefore approximated principal instability boundaries are given as follows.

$$
\frac{\theta}{2 \Omega}=\sqrt{1 \pm \mu}
$$

In Fig. 3, principal dynamic instability boundaries are presented in parameter plane $(\theta /(2 \Omega), \mu)$. Instability boundaries (14) can be rewritten as different expression (15) by substituting Eq. (8) into Eq. (14). 


$$
\frac{\theta}{2 \omega_{1}}=\sqrt{1-\frac{P_{0}}{P_{1}^{c r}} \pm \frac{P_{t}}{2 P_{1}^{c r}}}
$$

In expression (15), one may easily notice that unstable excitation frequency $\theta$ is decreased according to increasing the static loading $P_{0}$, and unstable region is expanded according to increasing the amplitude of dynamic load $P_{t}$. It is noted that unstable excitation frequency $\theta$ becomes zero, if amplitude of dynamic amplitude $P_{t}$ becomes zero and static load $P_{0}$ becomes fundamental Euler buckling load $P_{1}^{c r}$. This is compatible with the fact that structure is statically unstable under fundamental Euler buckling load. Additionally, it is remarked that unstable excitation frequency $\theta$ becomes similar to twice the fundamental natural frequency $\omega_{1}$ if amplitude of dynamic load $P_{t}$ is small and static load $P_{0}$ is zero.

In case of general structures, unstable boundary could be obtained by solving eigenvalue problem as shown in Eq. (16).

$$
\left|\mathbf{K}+\mathbf{K}_{g}^{(s)} \pm \frac{\beta}{2} \mathbf{K}_{g}^{(d)}-\frac{\theta^{2}}{4} \mathbf{M}\right|=0
$$

where $\mathbf{K}$ and $\mathbf{M}$ are stiffness and mass matrices, respectively. $\mathbf{K}_{g}^{(s)}$ and $\mathbf{K}_{g}^{(d)}$ are geometric stiffness matrices for static and dynamic loadings, respectively. $\beta$ and $\theta$ are dynamic load scale factor and frequency of dynamic loading, respectively. For detail discussion, one can see reference (Ahn and Ruzzene, 2006; Byun, et. al, 2011).

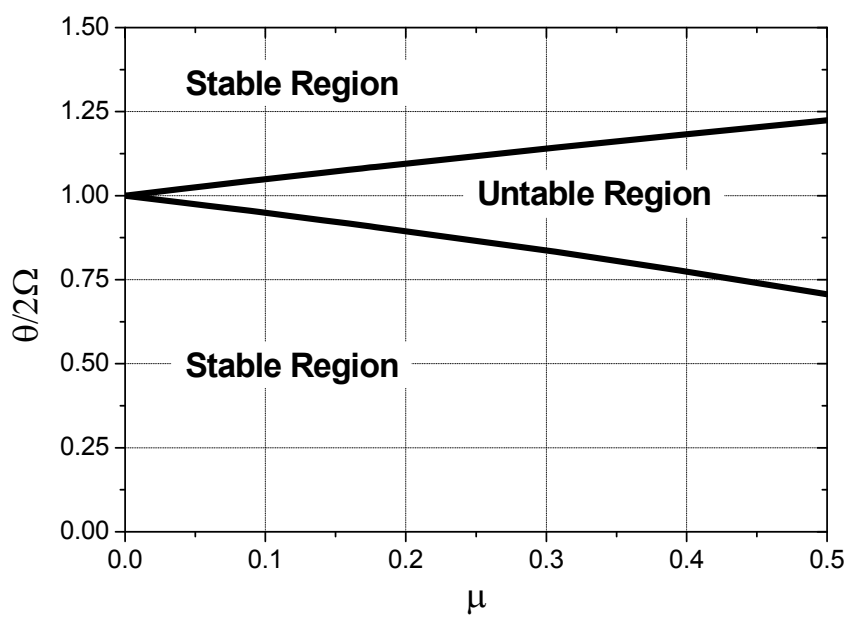

Fig. 3 Principal instability region in parameter plane $(\theta /(2 \Omega), \mu)$.

\section{DYNAMIC BUCKLING TEST SYSTEM}

In Fig. 4 and Fig. 5, layout of dynamic buckling test system is presented. Dynamic buckling test system consists of four parts. Those are structural frame, dynamic load generator, mechanism for static loading, and measurement system. In the following subsections, major four parts are described in detail.

\section{Structural frame and fixtures for dynamic buckling tests}

In this work, structural frame for dynamic buckling tests are constructed by using rectangular aluminum profiles. To prevent loosening of connecting bolts, nylon nuts are utilized. By using the profiles, structural frame for test system can be easily modified and withstand various loading conditions according to experimental requirements.

Further, structural frame has been built on heavy surface plate in order to isolate the test system from other undesirable external vibrations. Through the isolation, reliable test results could be obtained. 


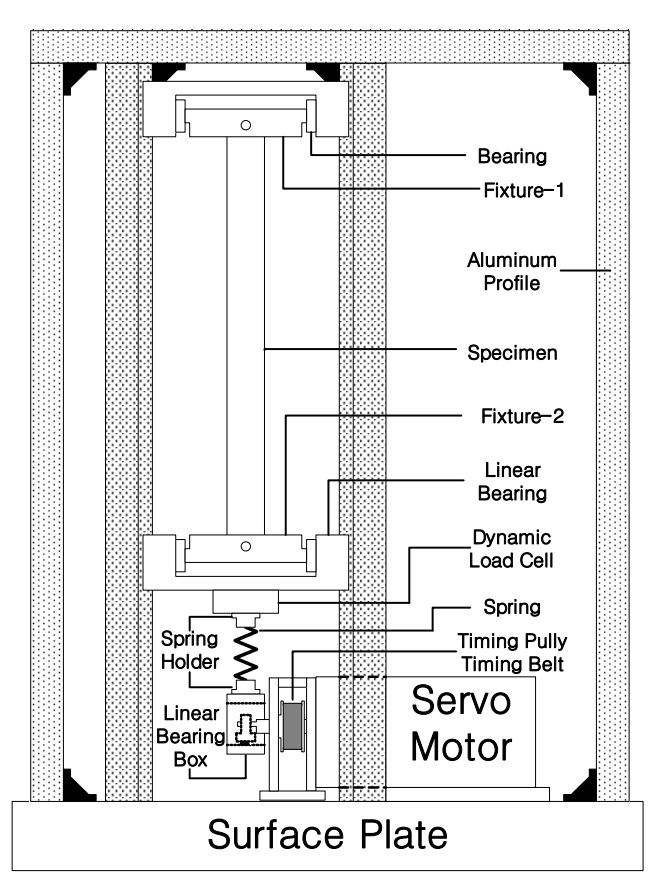

Fig. 4 Layout of dynamic buckling test system (front view).

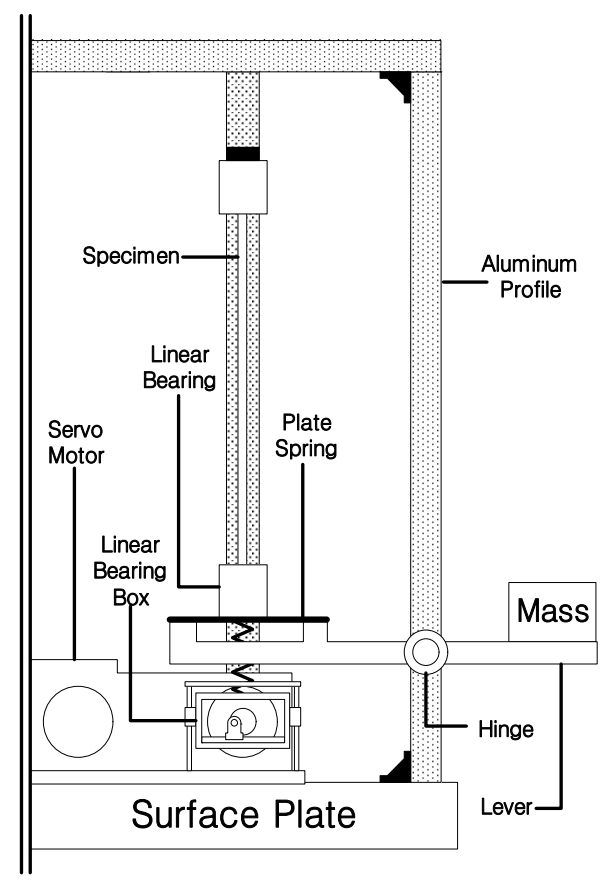

Fig. 5 Layout of dynamic buckling test system (side view).

For safety reason, the structural frame for dynamic buckling test system is covered by polycarbonate plates with $20 \mathrm{~mm}$ thickness. Due to the ductility of polycarbonate plates, it can be used as an efficient shield for small-size metallic debris from unexpected accidents. Furthermore, wire-mesh with $4 \mathrm{~mm}$ thickness wire is utilized to protect researchers from mid or large size fragments which may be separated from the test system under excessive dynamic buckling motion. Additionally, pieces of wire-mesh are connected by shackle to improve the impact resistance of protection system. Through this wire-mesh system, one can efficiently convert the kinetic energy of fragments into kinetic energy of wire-mesh as well as deformation energy.

For implementation of boundary conditions for specimens in dynamic buckling tests, two kinds of fixture systems are manufactured. One is pin-joint boundary condition fixture and the other is clamped boundary condition fixture. In case of pinjoint fixture, high speed rotational bearing is utilized to minimize the rotational friction. Fixture-1 at the top is installed to be fixed in vertical direction. Fixture-2 at bottom-side is installed by using linear bearing in order to move freely in vertical direction. At the bottom of fixture-2, load generator and dynamic load cell are attached to enforce and measure the applied load, respectively. Those parts are described in the following subsections in detail. Fig. 6 shows structural frame and protection wiremesh built on heavy surface plate for dynamic buckling tests.

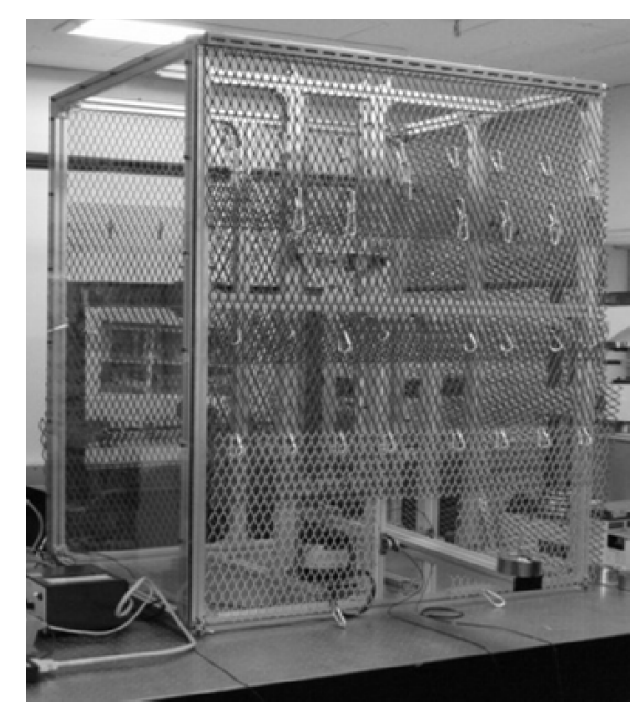

Fig. 6 Structural frame and protection wire-mesh built on heavy surface plate for dynamic buckling tests. 


\section{Dynamic load generator}

As a dynamic load generator, one may consider electromagnetic exciter (McConnell, 1995) which is usually adopted in vibration test. However, in dynamic buckling test, dynamic load should be delivered to specimen in axial direction of which stiffness is very large. Due to the reason, displacement becomes small in axial direction even though relatively large force is applied in dynamic buckling test. If one uses conventional electromagnetic exciter in this case, electromagnetic energy cannot be fully transformed to kinetic energy since the motion of armature of exciter is constrained in axial direction. As a result, large amount of electromagnetic energy is transformed to heat energy and increases the temperature of internal devices of exciter. Therefore, it may lead to fatal failure of electromagnetic exciter. Because of the reason, dynamic load generator is developed by using spring and servo motor in this work.

Fig. 7 shows the developed power delivery system made of pulley, timing belt, and servo motor. To change the rotational motion of pulley into translational motion, a bearing box is developed and attached to the bottom of coil spring as shown in Fig. 8. The bearing box is composed of rotational and vertical/horizontal linear bearing units. Through the device, kinematically exact sinusoidal motion in vertical (axial) direction can be obtained. Kinematical motion of bearing box is sketched in Fig. 9.

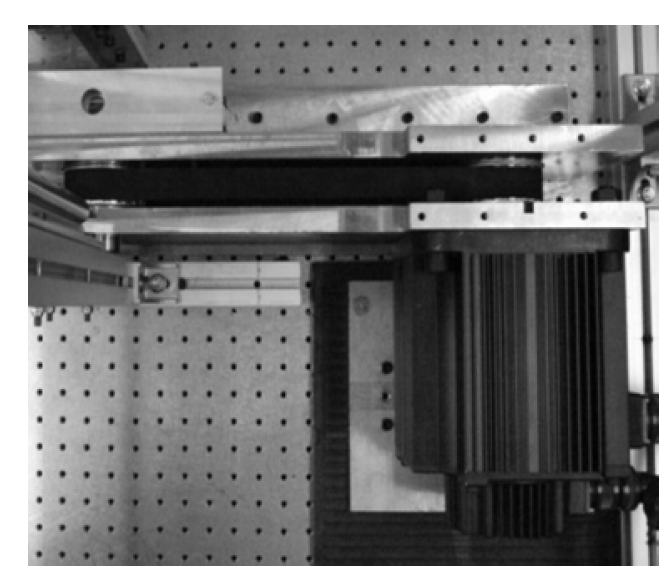

Fig. 7 Power delivery system made of pulley, timing belt, and servo motor.

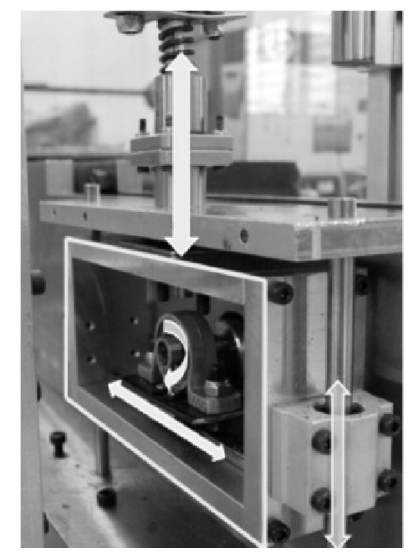

Fig. 8 Bearing box to change rotational motion to translational motion.
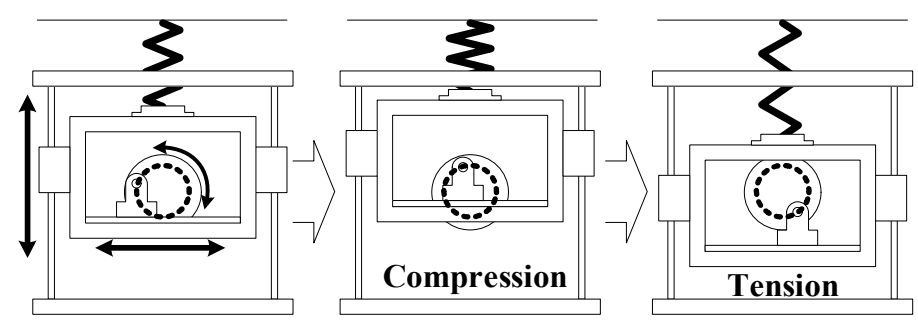

Fig. 9 Kinematic motion of bearing box.

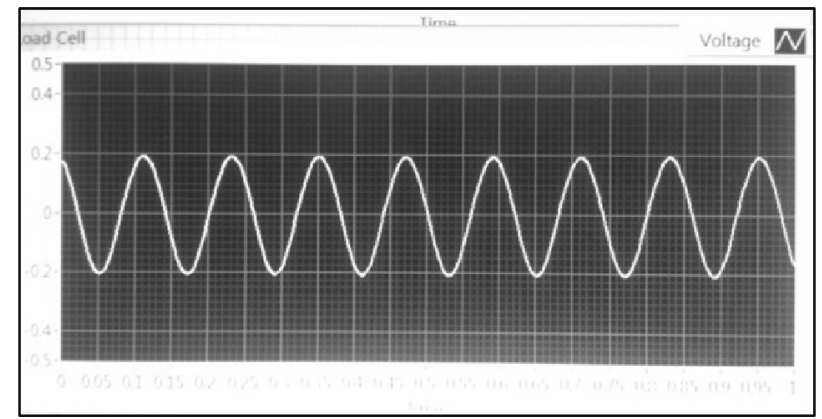

Fig. 10 Dynamic load measured by dynamic load cell. 
As shown in Fig. 9, bearing box moves in vertical direction according to rotational motion of pulley, and coil spring is dynamically compressed and stretched. As a result, one can apply nearly sinusoidal load to the specimen through the dynamically deformed coil spring. Amplitude of dynamic axial load can be changed by adopting various coil springs with different length and stiffness. Additionally, for the purpose of operating servo motor with uniform angular velocity, noise filter is utilized to eliminate input noise of AC power. Sinusoidal dynamic load, measured by dynamic load cell, is presented in Fig. 10.

\section{Mechanism for static loading}

In this work, static load is applied to the specimen by using lever principle. The magnitude of load applied to the specimen can be controlled by the mass and position of balance weight. To deliver the static load, generated by lever system, to the specimen, plate spring is utilized as shown in Fig. 5.
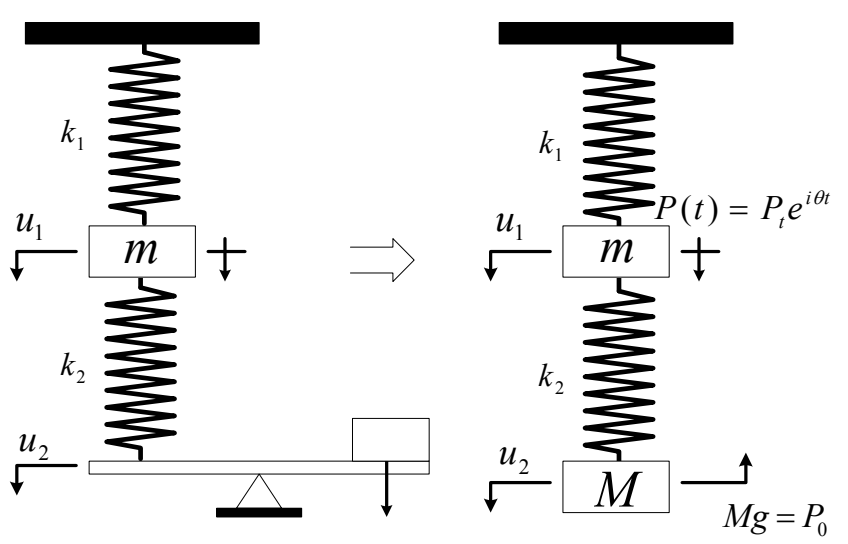

Fig. 11 Idealized dynamic models with lever system.

In left-hand side of Fig. 11, idealized dynamic model with lever system is presented. Its simplified model is presented in right-hand side of Fig. 11. In case of delivering static load through lever system, experiments may be disturbed because of dynamic interference between the specimen and lever system. To identify the dynamic interference, theoretical investigation is carried out. For the simplified dynamic model presented in right-hand side of Fig. 11, the equation of motion can be written in matrix form as follows.

$$
\left[\begin{array}{cc}
m & 0 \\
0 & M
\end{array}\right]\left\{\begin{array}{l}
\ddot{u}_{1} \\
\ddot{u}_{2}
\end{array}\right\}+\left[\begin{array}{cc}
k_{1}+k_{2} & -k_{2} \\
-k_{2} & k_{2}
\end{array}\right]\left\{\begin{array}{l}
u_{1} \\
u_{2}
\end{array}\right\}=\left\{\begin{array}{c}
P_{t} e^{i \theta t} \\
-P_{0}
\end{array}\right\}
$$

where $m$ and $M$ denote each mass. $k_{i}(i=1,2)$ and $u_{i}(i=1,2)$ correspond to spring constants and displacements, respectively. $P_{t} e^{i \theta t}$ is external dynamic load.

Let us assume the steady state solution as the form of (18).

$$
\left\{\begin{array}{l}
u_{1} \\
u_{2}
\end{array}\right\}=e^{i \theta t}\left\{\begin{array}{l}
X_{1} \\
X_{2}
\end{array}\right\}+\left\{\begin{array}{l}
C_{1} \\
C_{2}
\end{array}\right\}
$$

Then substituting expression (18) into (17) yields

$$
-\theta^{2} e^{i \theta t}\left[\begin{array}{cc}
m & 0 \\
0 & M
\end{array}\right]\left\{\begin{array}{l}
X_{1} \\
X_{2}
\end{array}\right\}+e^{i \theta t}\left[\begin{array}{cc}
k_{1}+k_{2} & -k_{2} \\
-k_{2} & k_{2}
\end{array}\right]\left\{\begin{array}{l}
X_{1} \\
X_{2}
\end{array}\right\}+\left[\begin{array}{cc}
k_{1}+k_{2} & -k_{2} \\
-k_{2} & k_{2}
\end{array}\right]\left\{\begin{array}{l}
C_{1} \\
C_{2}
\end{array}\right\}=e^{i \theta t}\left\{\begin{array}{c}
P_{t} e^{i \theta t} \\
0
\end{array}\right\}+\left\{\begin{array}{c}
0 \\
-P_{0}
\end{array}\right\}
$$


Since the coefficients of harmonic function and constant function should be identically zero in order to satisfy Eq. (19), one can obtain the following coefficient vectors (20) for steady state solution.

$$
\begin{gathered}
\left\{\begin{array}{l}
C_{1} \\
C_{2}
\end{array}\right\}=\frac{1}{k_{1} k_{2}}\left\{\begin{array}{c}
-k_{2} P_{0} \\
-\left(k_{1}+k_{2}\right) P_{0}
\end{array}\right\} \\
\left\{\begin{array}{l}
X_{1} \\
X_{2}
\end{array}\right\}=\frac{1}{\left(k_{1} k_{2}-\left(k_{1}+k_{2}\right) M \theta^{2}-k_{2} m \theta^{2} m M \theta^{4}\right)}\left\{\begin{array}{c}
\left(k_{2}-M \theta^{2}\right) P_{t} \\
k_{2} P_{t}
\end{array}\right\}
\end{gathered}
$$

On the other hand, the ideal system with no interference with lever system can be modeled as presented in Fig. 12, and its equation of motion can be written as

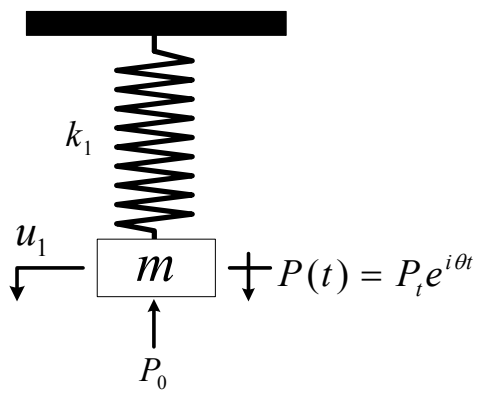

Fig. 12 Ideal model with no interference with lever system.

$$
m \ddot{u}_{1}+k_{1} u_{1}=-P_{0}+P_{t} e^{i \theta t}
$$

Comparing the first equation of (17) with Eq. (21), it is noticed that one can attain vibration isolation with no influence of lever system, if condition (22) is satisfied.

$$
k_{2}\left(u_{1}-u_{2}\right) \cong P_{0}
$$

By using steady state solution (18) with (20), condition denoted in (22) can be rewritten as follows.

$$
P_{0} \cong k_{2}\left(e^{i \theta t}\left(X_{1}-X_{2}\right)+C_{1}-C_{2}\right)=A+P_{0}, \quad A=k_{2} e^{i \theta t}\left(\frac{-M \theta^{2} P_{t}}{k_{1} k_{2}-\left(k_{1}+k_{2}\right) M \theta^{2}-k_{2} m \theta^{2} m M \theta^{4}}\right)
$$

Therefore $A$ in Eq. (23) should be minimized in order to decrease the dynamic influence of lever system. Further, $A$ can be rewritten as Eq. (24) through some algebraic manipulations.

$$
A=\left(\frac{P_{t}}{1+B}\right) e^{i \theta t}
$$

where $B$ has the following form (25) under the definitions of $\omega_{1}^{2} \equiv\left(k_{1} / m\right)$ and $\omega_{2}{ }^{2} \equiv\left(k_{2} / M\right)$. 


$$
B=\frac{k_{1}}{k_{2}}\left[1-\left(\theta / \omega_{1}\right)^{2}\right]\left[1-\left(\omega_{2} / \theta\right)^{2}\right]
$$

Consequently, magnitude of $B$ should be increased in order to decrease the magnitude of $A$. Considering the practical situation where the excitation frequency $\theta$ is much smaller than the lowest natural frequency of specimen $\omega_{1}$ in axial direction, it is natural to assume that $\left(\theta / \omega_{1}\right)$ is negligible compared with 1 . Further, it is difficult to make lever system of which natural frequency is much higher than operational excitation frequency $\theta$, taking into account the mass of weight balance and stiffness of conventional materials. Therefore, it is highly difficult to make the term $\left(\omega_{2} / \theta\right)$ very large. Rather than that, it is easier to make lever system such that $\left(\omega_{2} / \theta\right)$ is much less than 1 . Therefore it can be known that if the following two conditions are satisfied at the same time, one can attain ideal condition, where lever system does not influence on the dynamic motion of specimen.

$$
\left\{\begin{array}{l}
\text { Condition } 1: k_{1}>>k_{2} \\
\text { Condition } 2: \theta>>\omega_{2}
\end{array}\right.
$$

Condition 1 implies that the stiffness of plate spring is very small compared with axial stiffness of specimen. Condition 2 could be interpreted as the excitation frequency is larger than the natural frequency of lever system with plate spring.

Based on the above theoretical observation, stiffness of plate spring is determined in order to deliver the static load with minimal dynamic influence on the specimen. In this work, the stiffness of plate spring is adjusted by changing its width and thickness, considering the fact that plate spring should be able to withstand the force generated by lever system.

\section{Measurement of load and response}

To identify whether accurate dynamic load is applied to the specimen or not, dynamic load is measured by dynamic load cell of which operational frequency range is up to $2 \mathrm{kHz}$. Dynamic load cell is installed at the bottom fixture of specimen.

To calibrate dynamic load cell, lumped mass ' $m$ ' is attached to the load cell as shown in Fig. 13. And under its dynamic translational motion with acceleration ' $a$ ', dynamic load is measured. Through the fact that measured dynamic load should be the same as ' $m \times a$ ', dynamic load cell is calibrated. As mentioned before, Fig. 10 shows the measured signal for dynamic sinusoidal load, in which noise is successfully prevented. Additionally, static load delivered to the specimen is measured through load cell attached to the bottom of the fixture-2.
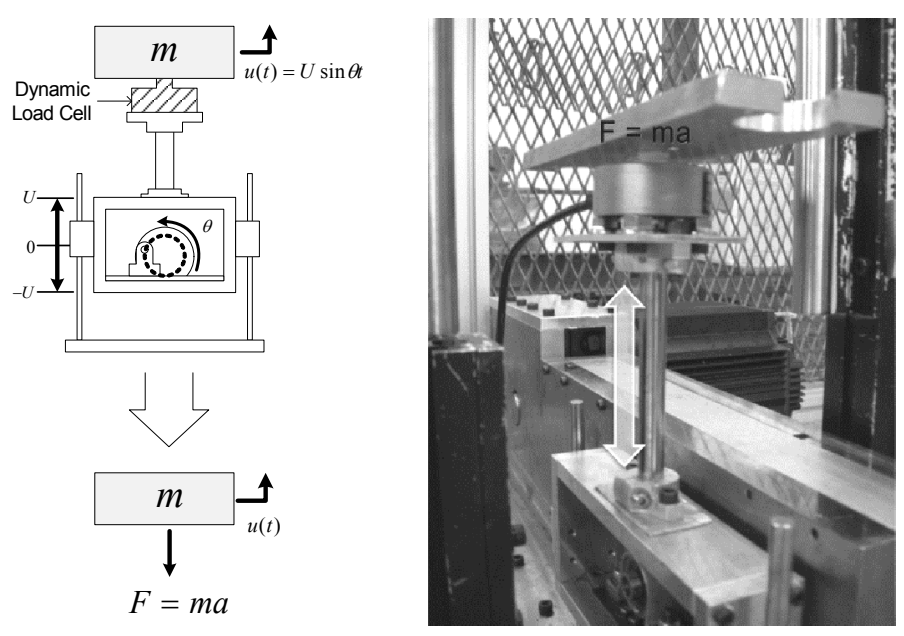

Fig. 13 Calibration of dynamic load cell.

In order to measure the response of specimen, piezoelectric accelerometer is attached to the position, where large transverse deflection occurs during dynamic buckling test. Voltage signal obtained from accelerometer is amplified via charge type am- 
plifier, and the amplified voltage signal is converted to digital signal by using USB type DAQ board. With the converted digital signal of acceleration, FFT analysis is carried out by LabVIEW software. Finally, unstable excitation frequency, which causes dynamic buckling (parametric resonance) of specimen, is determined through the frequency response as well as the time domain response. In Fig. 14, schematic of measurement system is presented.

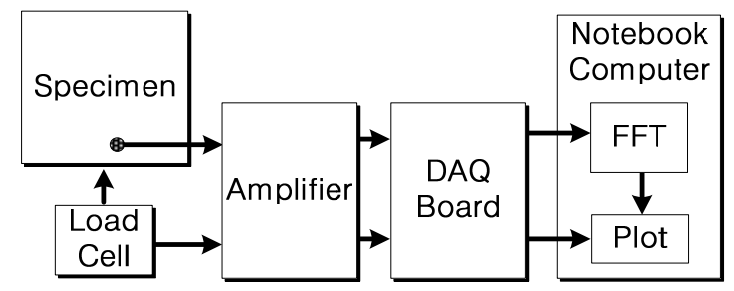

Fig. 14 Schematic of measurement system for load and response in dynamic buckling test.

\section{EXPERIMENTAL RESULTS}

By using the developed dynamic buckling test system, various tests have been carried out for beam, plate, and stiffened plate in order to identify the reliability of developed dynamic buckling test system as well as investigate the characteristics of dynamic buckling behavior of thin structures. In this work, dynamic buckling tests are carried out according to the following procedure.

(1) Determine the dimension and material of specimen.

(2) Manufacture the specimen and fixture.

(3) Measure the mass of fixture. It will be used in finite element analysis.

(4) Measure the dimension and mass of specimen. From the measured mass, determine the real density of specimen. It will be used in finite element analysis.

(5) Set up the specimen in dynamic buckling test system.

(6) Measure the fundamental natural frequency of specimen with fixture. It will be used in finite element analysis.

(7) Check load generation system and calibrate measurement system. Measure the static load.

(8) Excite the specimen, and measure the amplitude of dynamic load and acceleration of specimen.

(9) Investigate the measured time domain response and frequency response. (If the amplitude of time domain response becomes larger and peak frequency response is near the half of the excitation frequency, the excitation frequency is determined as unstable excitation frequency. If not, it is determined as stable excitation frequency)

(10) Check whether the excitation frequency is on the instability boundary where stability characteristics is changed from stable(unstable) motion to unstable(stable) motion.

(11) Carry out procedures (8-10) with increased excitation frequency.

To compare experimental results with finite element prediction, it is essential to make a finite element model which appropriately reflects the dynamic characteristics of specimen. For the purpose, elastic modulus is determined for computation such that natural frequency of finite element model becomes similar to the measured fundamental natural frequency of specimen with fixture, since there is uncertainty in the elastic modulus provided by manufacturer. Additionally, the fundamental buckling load of finite element model with the determined elastic modulus is utilized to plot instability region in parameter plane $(\theta /(2 \Omega), \mu)$.

\section{Beam structure}

For pin-jointed aluminum beam structure, dynamic buckling tests are carried out. Young's modulus is 69 GPa, and its Poisson's ratio is 0.33 . Density is $2,710 \mathrm{~kg} / \mathrm{m}^{3}$. Length of beam is $800 \mathrm{~mm}$. Width of beam is $30 \mathrm{~mm}$, and thickness is $3 \mathrm{~mm}$. 


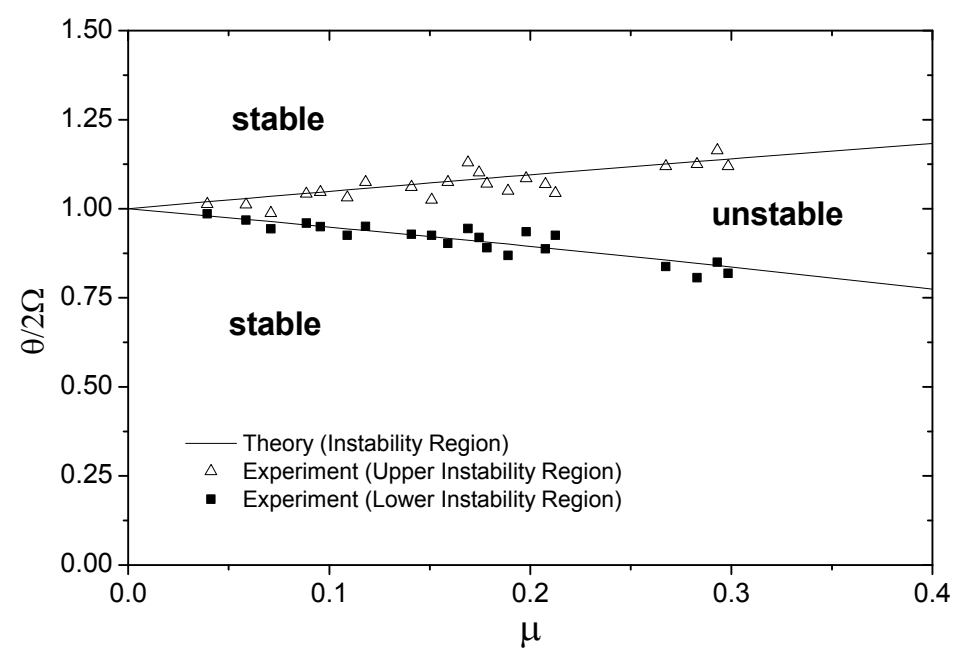

Fig. 15 Primary region of instability for pin-jointed beam structure $(800 \mathrm{~mm} \times 30 \mathrm{~mm} \times 3 \mathrm{~mm})$.
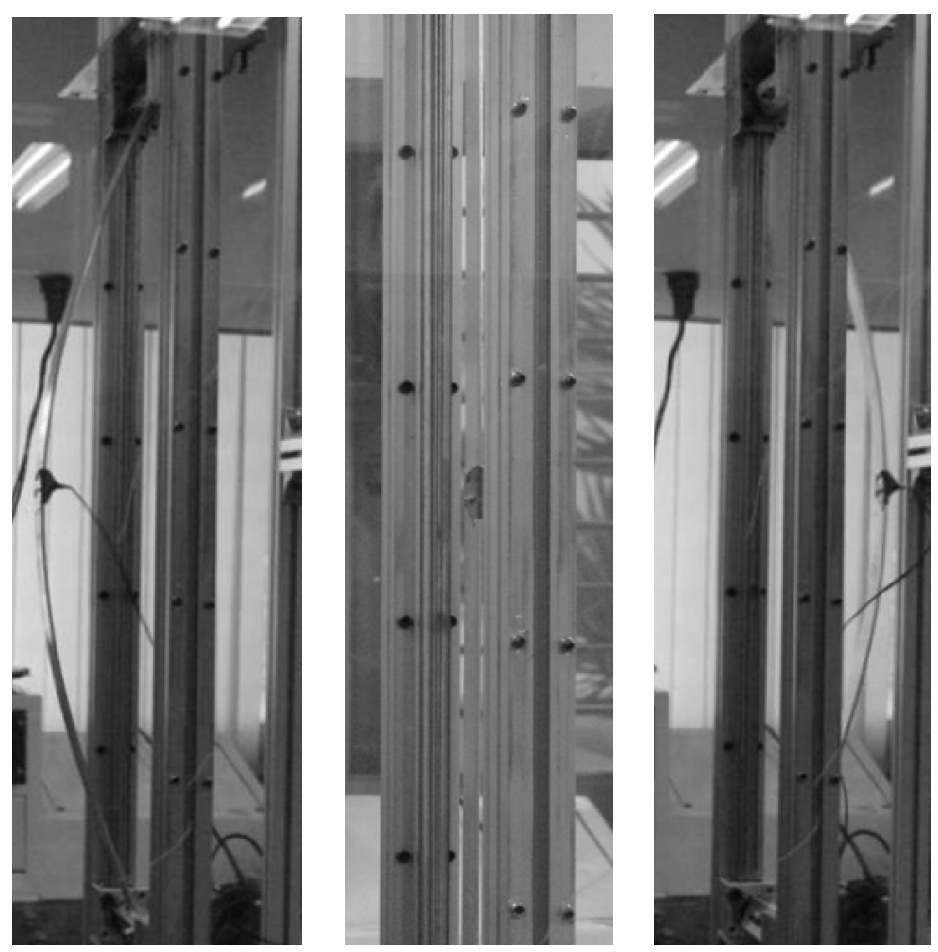

Fig. 16 Snapshot of dynamic buckling of pin-jointed beam structure $(800 \mathrm{~mm} \times 30 \mathrm{~mm} \times 3 \mathrm{~mm})$.

According to amplitude of dynamic load $P_{t}$ and static load $P_{0}$, dynamic buckling tests are carried out. Through the frequency response and time domain response obtained by the measurement system, unstable excitation frequency $\theta$ is determined. The results are plotted in aforementioned parameter plane as shown in Fig. 15, where normalized excitation frequency is denoted by $\theta /(2 \Omega)$, and load parameter is denoted by $\mu$.

From the results presented in Fig. 15, one can easily identify that the experimental results show good agreements with theoretical predictions. Maximum difference between experimental result and analytical solution is around 5\%. Additionally, one can observe that the region of instability is increased according to increasing load parameter $\mu$. From the results, it is confirmed that the current experimental approach is reasonable and the developed test system is reliable and useful to carry out dynamic buckling tests. Fig. 16 shows snapshot of dynamic buckling of pin-jointed beam structure which undergoes 12 $N$ static load and $26 \mathrm{~Hz}$ dynamic load with $22 \mathrm{~N}$ amplitude. 


\section{Plate and stiffened plate}

For plate and stiffened plate structures, dynamic buckling tests are performed. Dimensions of three specimens are presented in table 1. Specimen-1 is narrow plate, and specimen-2 is wide plate. Specimen-3 is narrow plate with stiffener as presented in right-hand side of Fig. 17. As presented in Fig. 17, two corners on the top surface are clamped, and static/dynamic loads are applied to the left corner at the bottom surface. Left corner at the bottom surface moves freely in vertical direction, while all of the other motions of left-bottom corner are constrained by clamped fixture. In table 2, material properties, characterized by vibration tests of specimens, are presented. The characterized properties are utilized to predict the dynamic buckling instability region by finite element analysis.

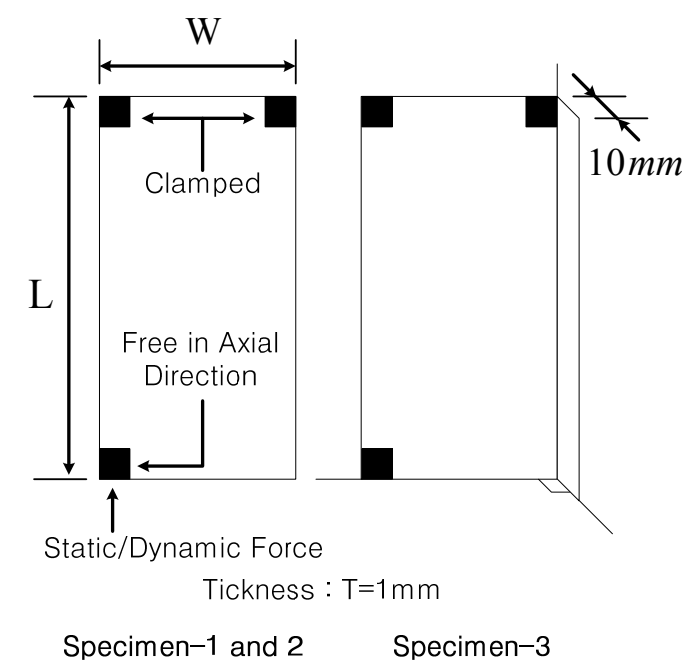

Fig. 17 Shapes and boundary conditions of specimens.

Table 1 Dimensions of plate and stiffened plate specimens.

\begin{tabular}{|c|c|c|c|c|}
\hline & Length $(L)$ & Width $(W)$ & Thickness $(T)$ & Height (Stiffener) \\
\hline Specimen-1 & $1000 \mathrm{~mm}$ & $250 \mathrm{~mm}$ & $1 \mathrm{~mm}$ & - \\
\hline Specimen-2 & $1000 \mathrm{~mm}$ & $500 \mathrm{~mm}$ & $1 \mathrm{~mm}$ & - \\
\hline Specimen-3 & $1000 \mathrm{~mm}$ & $250 \mathrm{~mm}$ & $1 \mathrm{~mm}$ & $10 \mathrm{~mm}$ \\
\hline
\end{tabular}

Table 2 Material properties used for dynamic buckling prediction by finite element analysis.

\begin{tabular}{|c|c|c|c|}
\hline & Density & Young's modulus & Poisson's ratio \\
\hline Specimen-1 & $2678.4 \mathrm{~kg} / \mathrm{m}^{3}$ & $63.3 \mathrm{Gpa}$ & 0.33 \\
\hline Specimen-2 & $2672.0 \mathrm{~kg} / \mathrm{m}^{3}$ & $54.1 \mathrm{Gpa}$ & 0.33 \\
\hline Specimen-3 & $2696.7 \mathrm{~kg} / \mathrm{m}^{3}$ & $62.4 \mathrm{Gpa}$ & 0.33 \\
\hline
\end{tabular}

For specimen-1 (narrow plate), dynamic buckling tests are carried out under various loading conditions according to aforementioned procedures. The experimental results are converted to normalized parameters $(\theta /(2 \Omega), \mu)$ and presented in Fig. 18 . Here the fundamental natural frequency $\omega_{1}$ and fundamental buckling load $P_{1}^{c r}$ of plate specimen-1 are utilized to calculate $\theta /(2 \Omega)$ and $\mu$ defined by Eq. (8). From the results, one can notice that the region of instability is increased according to increasing load parameter $\mu$ like beam structures. Additionally, it can be observed that instability boundary predicted by inhouse finite element program is similar to experimental results.

To observe the effect of width, dynamic buckling tests are carried out for specimen-2 (wide plate), and the results are compared with the results obtained for specimen-1. In this test, static load is not applied through the lever system. Therefore 
only the weight $(13.13 \mathrm{~N})$ of bottom fixture- 2 is considered as static load $P_{0}$ in finite element analysis. In the results, excitation frequencies $\theta$ on the instability boundaries are plotted according to the amplitude $P_{t}$ of dynamic load. The results show that the unstable region of excitation frequency is lowered, compared with narrow plate. From the result, it can be known that flexible structures with low fundamental natural frequency such as wide plate $(1,000 \mathrm{~mm} \times 500 \mathrm{~mm} \times 1 \mathrm{~mm})$ becomes unstable in lower excitation frequencies compared with stiff structures with higher fundamental natural frequency like narrow plate $(1,000 \mathrm{~mm} \times 250 \mathrm{~mm} \times 1 \mathrm{~mm})$.

To investigate effect of stiffness of structures further, dynamic buckling test is carried out for stiffened plate (specimen-3). Like the test in Fig. 19, static load is not applied through the lever system to observe the basic effect of stiffener on dynamic buckling behavior. For finite element computation, static load caused by weight of bottom fixture- 2 is considered. From the dynamic buckling test, the obtained excitation frequencies $\theta$ on the instability boundaries are plotted according to the amplitude $P_{t}$ of dynamic load in Fig. 20. In case of stiffened plate, there is larger possibility of imperfection compared with flat plate. It seems that the deviation between experimental result and finite element prediction for stiffened plate is caused by this kind of imperfection. However, the tendency of dynamic buckling of stiffened plate is compatible with finite element prediction. From the experimental results, one can identify that the region of instability is raised by using stiffener although other dimensions are not changed.

The result reveals that one can use stiffener in design of high-speed underwater vehicle to avoid fatal dynamic buckling in given loading environment. Additionally, it is known that in-house finite element program for dynamic buckling prediction is useful in design of structures to avoid fatal dynamic buckling phenomena. As an example, snapshot of dynamic buckling of clamped plate is presented in Fig. 21.

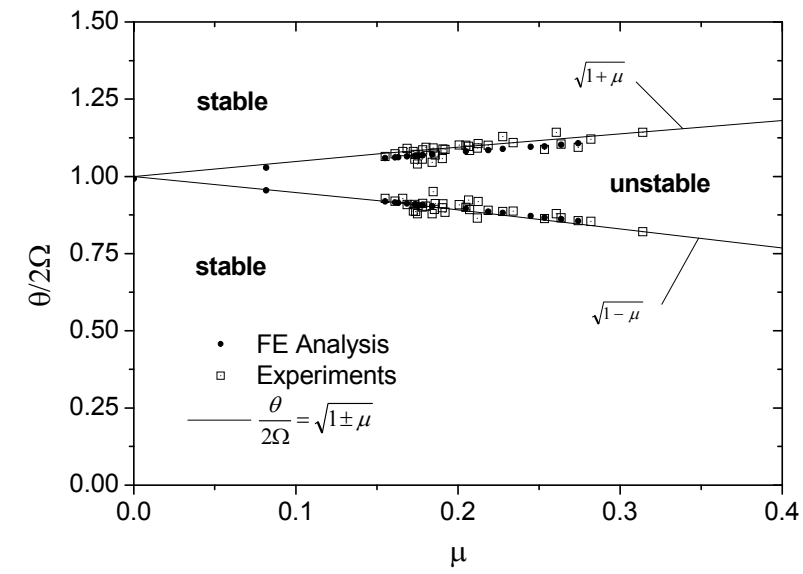

Fig. 18 Primary region of instability for specimen-1 plotted in parameter plane.

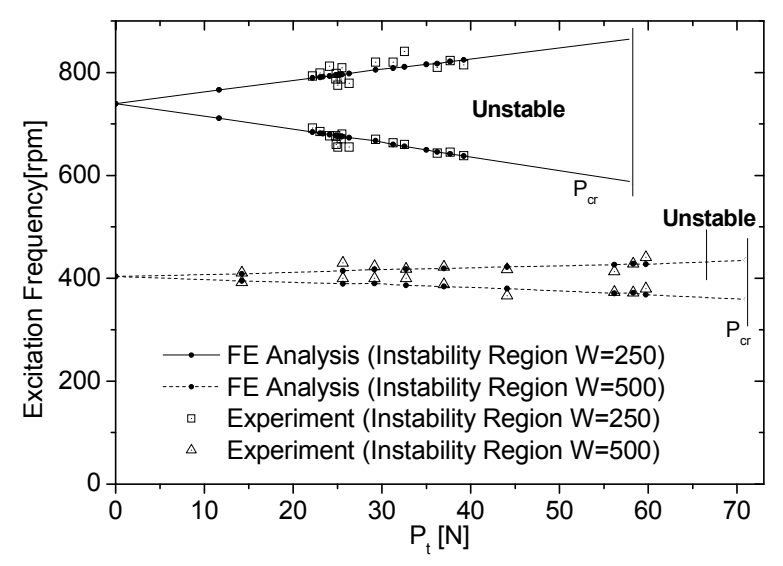

Fig. 19 Effect of width of plate on primary region of instability (specimen-2 (width $500 \mathrm{~mm}$ ) vs. specimen-1 (width $250 \mathrm{~mm}$ )).

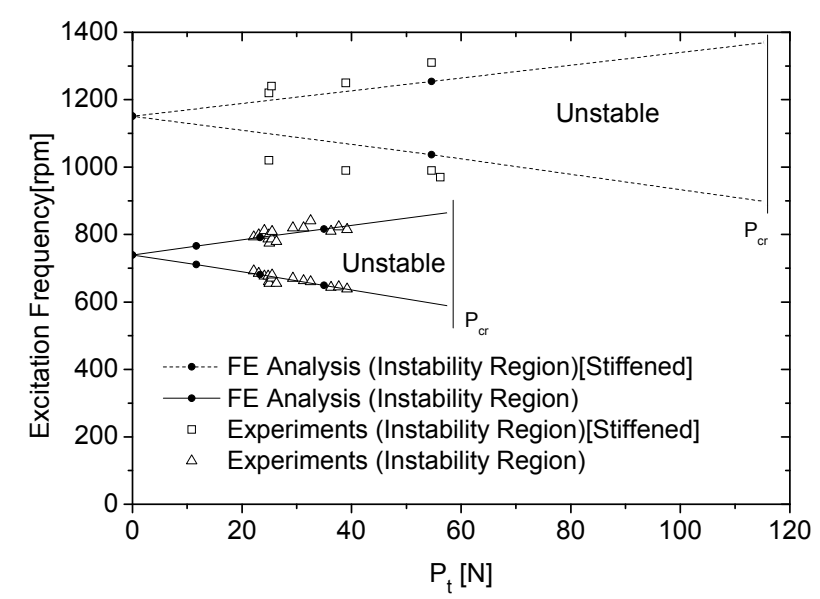

Fig. 20 Effect of stiffener on primary region of instability (specimen-3 (10 $\mathrm{mm}$ stiffener) vs. specimen-1 (no stiffener)). 

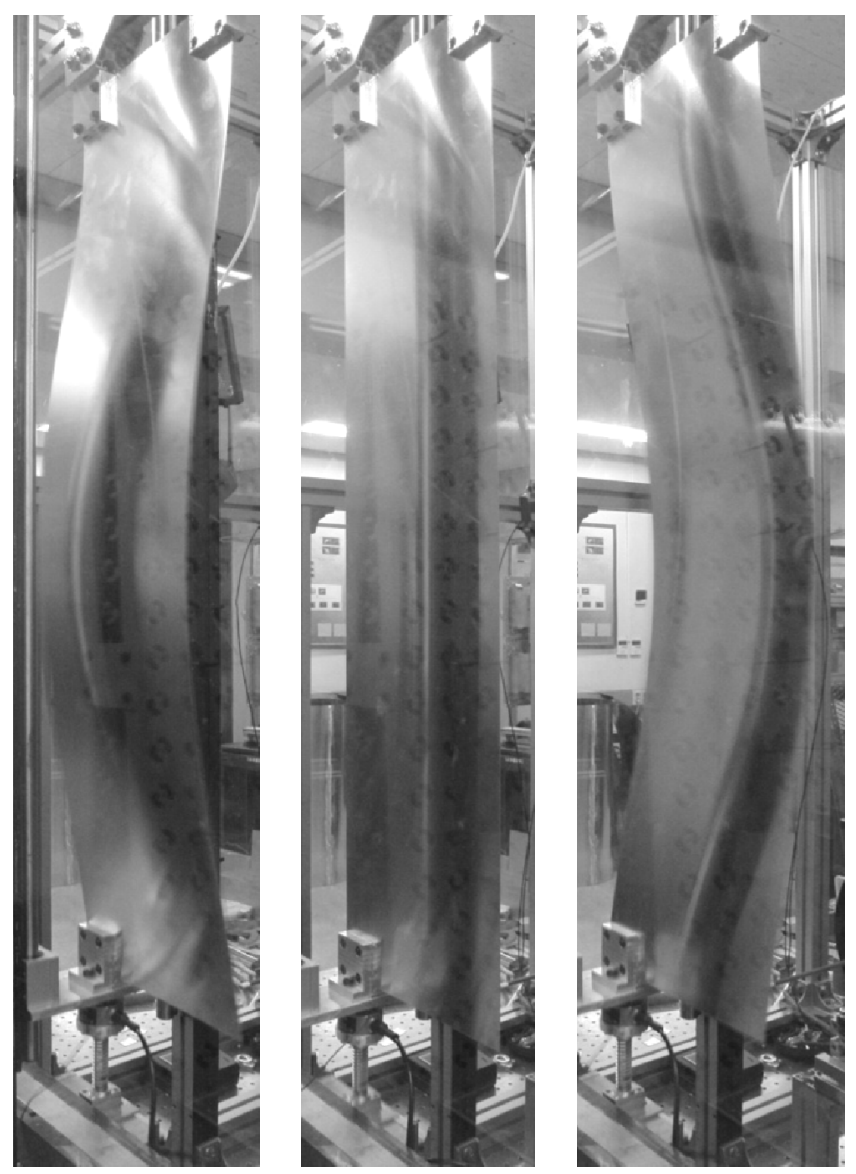

Fig. 21 Snapshot of dynamic buckling of clamed plate (static load of fixture weight and $12 \mathrm{~Hz}$ dynamic load with $22 \mathrm{~N}$ amplitude).

\section{SUMMARY AND CONCLUSIONS}

Dynamic buckling is one of the dynamic instability phenomena which may cause catastrophic failure of structure, when the structure undergoes compressive dynamic loads. Therefore, it is essential to investigate the dynamic buckling characteristics and devise design methodologies to avoid such fatal structural instability phenomena, especially when the design target is supposed to be utilized in high compressive dynamic loading environment. High-speed underwater vehicle is one such example.

In the line of thought, experimental study on dynamic buckling phenomena is carried out in this work. Major effort is focused on the development of reliable dynamic buckling test system and experimental investigation of characteristics of dynamic buckling.

From the experimental results, it is observed that unstable frequency region is increased according to increasing the amplitude of dynamic load. Additionally, from the comparison between wide plate and narrow plate, it is known that dynamic buckling region is lowered when the fundamental natural frequency of structure becomes smaller. Also the experiment with stiffened plate reveals that one can avoid dynamic buckling by adopting stiffener in design of structures under compressive loading environment.

Through the experiments and crosscheck with analytical and computational results, it is confirmed that the developed dynamic buckling test system is reliable. Additionally current study shows a lot of possibility that the current approach to construct dynamic buckling test system can be also adopted in constructing real scale dynamic buckling test system.

\section{ACKNOWLEDGEMENTS}

This work was supported by Defense Acquisition Program Administration and Agency for Defense Development under the contract number 09-01-08-25, and by Inha University Research Grant. 


\section{REFERENCES}

Ahn, S.S. and Ruzzene, M., 2006. Optimal design of cylindrical shells for enhanced buckling stability: application to supercavitating underwater vehicles. Finite Elements in Analysis and Design, 42(11), pp.967-976.

Bolotin, V.V., 1964. The dynamic stability of elastic systems. London : Holden-Day, Inc.

Byun, W., Kim, M.K., Park, K.J., Kim, S.J, Chung, M., Cho, J.Y. and Park, S.-H., 2011. Buckling analysis and optimal structural design of supercavitating vehicles using fintie element technology. Internationl Journal of Naval Architecture \& Ocean Engineering, 3(4), pp. 274-285.

Chang, B.C., 2008. On the parametric rolling of ships using a numerical simulation method. Ocean Engineering, 35(5-6), pp.447-457.

Chen, C.-C. and Yeh, M.-K., 2001. Parametric instability of a beam under electromagnetic excitation. Journal of Sound and Vibration, 240(4), pp.747-764.

Deolasi, P.J. and Datta, P.K., 1997. Experiments on the parametric vibration response of plates under tensile loading. Experimental Mechanics, 37(1), pp.56-61.

Hargrove, J., 2004. Supercavitation and aerospace technology in the development of high-speed underwater vehicles. Proceeding of 42nd AIAA aerospace sciences meeting. Reno, Nevada, USA, AIAA-2004-0130.

Iwatsubo, T. and Saigo, M., 1974. Simple and combination resonances of columns under periodic axial loads. Journal of Sound and Vibration, 33(2), pp.211-221.

Langthjem, M.A. and Sugiyama, Y., 2000. Dynamic stability of columns subjected to follower loads: a survey. Journal of Sound and Vibration, 238(5), pp.809-851.

McConnell, K.G., 1995. Vibration testing, theory and practice. New York: John Wiley \& Sons.

Meirovitch, L., 1998. Methods of analytical dynamics. New York: McGraw-Hill.

Park, S.-H. and Kim, J.-H., 2002. Dynamic stability of a stiff-edged cylindrical shell subjected to a follower force. Computers and Structures, 80(3-4), pp.227-233.

Popov, A.A., 2003. Parametric resonance in cylindrical shells: a case study in the nonlinear vibration of structural shells. Engineering Structures, 25(6), pp.798-799.

Savchenko, Y., 2001. Supercavitation - problems and perspectives. Proceedings of fourth international symposium on cavitation. Pasadena, CA, USA.

Vanek, B., Bokor, J., Balas, G.J. and Arndt, R.E.A., 2007. Longitudinal motion control of a high-speed supercavitation vehicle. Journal of Vibration and Control, 13(2), pp.159-184.

Vlasenko, Y.D., 2003. Experimental investigation of supercavitation flow regimes at subsonic and transonic speeds. Proceedings of fifth international symposium on cavitation. Osaka, Japan. 